\title{
OPTIMAL PLANE CHANGE BY LOW AERODYNAMIC FORCES
}

\author{
Nguyen X. Vinh* \\ The University of Michigan \\ Ann Arbor, Michigan \\ and \\ Der-Ming $\mathrm{Ma}^{\dagger}$ \\ Chung Shan Institute of Science and Technology \\ Lung Tan, Taiwan, R.O.C
}

\begin{abstract}
This paper presents the exact dimensionless equations of motion and the necessary conditions for the computation of the optimal trajectories of a hypervelocity vehicle flying through a non-rotating spherical planetary atmosphere. Solution is then presented for the case where starting with a super-circular entry speed $V_{0}>V_{c}$, optimal aerodynamic maneuver, in terms of the lift coefficient and the bank angle, is performed leading to an exit circular speed $V_{f}=V_{c}$ while maximizing the plane change $I_{f}$. It is shown that there are two types of maneuvers with nearly identical plane change. In the hard maneuver, the vechicle is pulled down to low altitude for aerodynamic plane change before exit at the prescribed final speed. In the slow maneuver which is described in detail in this paper, the vehicle remains in orbital flight with a small incremental plane change during each passage through the perigee. This maneuver requires several revolutions, and the technique for computation is similar to that in the problem of contraction of orbit.
\end{abstract}

\section{Nomenclature}

$\begin{array}{ll}a & =\text { semimajor axis } \\ B & \text { =ballistic coefficient defined in Eq. (12) } \\ C & =\text { vertical component of normalized lift } \\ & \text { coefficient } \\ C_{D} & =\text { drag coefficient } \\ C_{D_{0}} & \text { =zero-lift drag coefficient } \\ C_{L} & =\text { lift coefficient } \\ c_{i} & =\text { constants of integration } \\ E & =\text { lift-to-drag ratio } \\ \mathrm{e} & =\text { natual exponential } \\ e & =\text { eccentricity } \\ g & =\text { gravity acceleration } \\ h & =\text { dimensionless altitude } \\ I & \text { =inclination angle } \\ K & \text { =induced drag parameter }\end{array}$

*Professor of Aerospace Engineering. Member AIAA. †Associate Research Scientist.

Copyright (C) 1990 American Institute of Aeronautics and Astronautics, Inc. All rights reserved.

$\begin{array}{ll}k_{i} & =\text { constants of integration } \\ m & =\text { mass } \\ r & =\text { radial distance } \\ S & =\text { lateral component of normalized lift } \\ & \text { coefficient } \\ s & =\text { dimensionless arc length } \\ t & =\text { time } \\ u & =\text { dimensioless velocity squared } \\ V & =\text { velocity } \\ \beta & =\text { inverse of scale height } \\ \gamma & =\text { flight path angle } \\ \epsilon & =\text { parameter defined in Eq. (12) } \\ \theta & =\text { longitude } \\ \lambda & =\text { normalized lift coefficient } \\ \rho & =\text { air density } \\ \sigma & =\text { bank angle } \\ \phi & =\text { latitude } \\ \psi & =\text { heading }\end{array}$

Superscript

* $\quad=\underset{\text { ratio }}{\text { condition at maximum lift-to-drag }}$

Subscripts
$f \quad=$ final value
$0 \quad=$ for $g, r$ and $\rho$, value at reference point
$0 \quad=$ for $s, u$ and $\gamma$, initial value

\section{Introduction}

In recent years, there is a strong interest in the problem of optimal aeroassisted orbit transfer. A recent survey by Mease ${ }^{1}$ has provided an extensive list of references. It is known that the use of the atmospheric forces to perform the plane change during a transfer from orbit to orbit can significantly reduce the total fuel consumption as compared to a pure propulsive transfer. Most of the studies such as the one given in Ref. 2 concern the atmospheric maneuver in one single passage through the atmosphere. This type of maneuver is the hard maneuver and it encounters strong 
deceleration and heating rate. Another possibility is to keep the lifting vehicle in orbital flight with its perigee low enough such that during each passage through this point a small plane change can be achieved. This type of slow maneuver will be considered in this paper and it is shown that for a same speed depletion it provides the same plane change as compared to the hard maneuver.

\section{The Dimensionless Equations of Motion}

For the flight over a spherical, non-rotating planet of a non-thrusting, aerodynamic vehicle with the lift coefficient $C_{L}$ and the drag coefficient $C_{D}$, it is customary to use the equations of motion with the notation in Fig. 1. We have

$$
\begin{aligned}
\frac{d r}{d t} & =V \sin \gamma \\
\frac{d \theta}{d t} & =\frac{V \cos \gamma \cos \psi}{r \cos \phi}, \\
\frac{d \phi}{d t} & =\frac{V \cos \gamma \sin \psi}{r}, \\
\frac{d V}{d t} & =-\frac{\rho S C_{D} V^{2}}{2 m}-g \sin \gamma, \\
V \frac{d \gamma}{d t} & =\frac{\rho S C_{L} V^{2}}{2 m} \cos \sigma-\left(g-\frac{V^{2}}{r}\right) \cos \gamma, \\
V \frac{d \psi}{d t} & =\frac{\rho S C_{L} V^{2}}{2 m \cos \gamma} \sin \sigma-\frac{V^{2}}{r} \cos \gamma \cos \psi \tan \phi,
\end{aligned}
$$

where the bank angle $\sigma$ is defined as the angle between the local vertical plane containing the velocity and the plane containing the velocity and the aerodynamic force.

For continuity between atmospheric flight and flight in the vacuum, we define the dimensionless variables

$$
\begin{aligned}
u & =\frac{V^{2}}{g_{0} r_{0}}, \\
h & =\frac{r-r_{0}}{r_{0}}
\end{aligned}
$$

to represent the speed and the altitude variables and the dimensionless arc length

$$
s=\int_{0}^{t} \frac{V}{r} \cos \gamma d t
$$

to replace the time as the independent variable. The reference distance $r_{0}$ is the initial perigee distance. The drag polar used is the parabolic drag polar

$$
C_{D}=C_{D_{0}}+K C_{L}^{2}
$$

with the condition at maximum lift-to-drag ratio

$$
\begin{aligned}
C_{L} & =C_{L}^{*}=\sqrt{C_{D_{0}} / K}, \\
C_{D} & =C_{D}^{*}=2 C_{D_{0}}, \\
E^{*} & =C_{L}^{*} / C_{D}^{*} .
\end{aligned}
$$

The aerodynamic control can be modeled as the modulation of the vertical and the lateral component of the normalized lift coefficient $\lambda$

$$
C=\lambda \cos \sigma, \quad S=\lambda \sin \sigma,
$$

where

$$
\lambda=\frac{C_{L}}{C_{L}^{*}}
$$

Using a Newtonian gravitational field

$$
\frac{g}{g_{0}}=\frac{r_{0}^{2}}{r^{2}}
$$

and a locally exponential atmosphere

$$
\rho=\rho_{0} \mathrm{e}^{-\beta r_{0} h}=\rho_{0} \mathrm{e}^{-h / \epsilon}
$$

we obtain the universal equations for flight in both inside the atmosphere and in the vacuum:

$$
\begin{aligned}
& \frac{d h}{d s}=(1+h) \tan \gamma \\
& \frac{d \theta}{d s}=\frac{\cos \psi}{\cos \phi} \\
& \frac{d \phi}{d s}=\sin \psi \\
& \frac{d \psi}{d s}=\frac{B \lambda \sin \sigma(1+h) e^{-h / \epsilon}}{\cos ^{2} \gamma}-\cos \psi \tan \phi, \\
& \frac{d u}{d s}=-\frac{B(1+h) u\left(1+\lambda^{2}\right) e^{-h / \epsilon}}{E^{*} \cos \gamma}-\frac{2}{(1+h)} \tan \gamma, \\
& \frac{d \gamma}{d s}=\frac{B(1+h) \lambda \cos \sigma e^{-h / \epsilon}}{\cos \gamma}+1-\frac{1}{u(1+h)} .
\end{aligned}
$$

In the equations above, we have defined

$$
\epsilon=1 / \beta r_{0} \quad \text { and } \quad B=\frac{\rho_{0} S C_{L}^{*} r_{0}}{2 m} .
$$

In these equations the only physical characteristics of the vehicle are its maximum lift-to-drag ratio, $E^{*}$, and the ballistic coefficient $B$ specifying the starting flight altitude. We consider the case $E^{*}=1.5$ for the computation, and take a value $B$ low enough for the vehicle to stay in orbit for several revolutions. The value $1 / \epsilon=\beta r_{0}=900$ used is representative of the Earth's atmosphere. 
At this point, it is not difficult to apply the variational principle to system (11) and derive the necessary conditions for computing the optimal trajectory leading from

$$
(h, \theta, \phi, \psi, u, \gamma)=\left(0,0,0,0, u_{0}, \gamma_{0}\right)
$$

at the initial time $s_{0}=0$, to the final time $s_{f}=$ free such that

$$
(h, \theta, \phi, \psi, u, \gamma)=\left(0, \text { free }, \phi_{f}, \psi_{f}, u_{f}, \gamma_{f}\right)
$$

where $u_{f}$ and $\gamma_{f}$ are prescribed and $\phi_{f}$ and $\psi_{f}$ are such that

$$
J=\cos I_{f}=\cos \phi_{f} \cos \psi_{f}
$$

is minimized so that the plane change $I_{f}$ is a maximum.

This has been done in Ref. 4 and it is shown that the maneuver can be performed in one passage through the atmosphere using strong aerodynamic forces or by several passages with small plane change each time the vehicle passes through its perigee. Since in this case, the vehicle stays in orbital flight, the aerodynamic forces used are low. The numerical solution obtained by solving a two-point-boundary-value problem for long duration flight is tedious. We shall propose a simplified but accurate and fast scheme for the computation of the plane change.

\section{Approximate Aerodynamic Control}

Introducing the adjoint variables $p_{x}$, we form the Hamiltonian

$$
\begin{aligned}
H= & p_{h}(1+h) \tan \gamma+p_{\theta} \frac{\cos \psi}{\cos \phi}+p_{\phi} \sin \psi \\
& +p_{\psi}\left[\frac{B \lambda \sin \sigma(1+h) \mathrm{e}^{-h / \epsilon}}{\cos ^{2} \gamma}-\cos \psi \tan \phi\right] \\
& -p_{u}\left[\frac{B(1+h) u\left(1+\lambda^{2}\right) \mathrm{e}^{-h / \epsilon}}{E^{*} \cos \gamma}+\frac{2}{1+h} \tan \gamma\right] \\
& +p_{\gamma}\left[\frac{B(1+h) \lambda \cos \sigma \mathrm{e}^{-h / \epsilon}}{\cos \gamma}+1-\frac{1}{u(1+h)}\right]
\end{aligned}
$$

The maximization of the Hamiltonian with respect to the controls $\lambda$ and $\sigma$ leads to the optimal law

$$
C=\frac{E^{*} p_{\gamma}}{2 p_{u} u}, \quad S=\frac{E^{*} p_{\psi}}{2 p_{u} u \cos \gamma} .
$$

Along the optimal trajectory, the adjoint variable $p_{x}$, for any state variable $x$, satisfies the adjoint equation

$$
\frac{d p_{x}}{d s}=-\frac{\partial H}{\partial x} \text {. }
$$

It is known that the problem has the following integrals ${ }^{3}$

$$
\begin{aligned}
& H=c_{0}, \\
& p_{\theta}=c_{1}, \\
& p_{\phi}=c_{2} \sin \theta-c_{3} \cos \theta, \\
& p_{\psi}=c_{1} \sin \phi+\left(c_{2} \cos \theta+c_{3} \sin \theta\right) \cos \phi,
\end{aligned}
$$

where the $c_{i}$ are constants of integration. In this problem of optimal plane change, we are concerned with speed depletion and heading angle without prescribing the final arc length $s_{f}$ and final longitude $\theta_{f}$. Hence, by the transversality condition

$$
c_{0}=0, \quad c_{1}=0 .
$$

With the four integrals, only two of the remaining adjoint equations need to be integrated.

With the Hamiltonian integral $H=c_{0}$, the solutions for any two of the three adjoint variables $p_{h}, p_{u}$ and $p_{\gamma}$ are not known explicitly for atmospheric flight but for low aerodynamic forces, by neglecting the density $B e^{-h / \epsilon}$, they can be obtained approximately as follows.

With the atmospheric density neglected, the motion is Keplerian and from Eqs. (11), we have the integrals expressing constant energy and constant angular momentum

$$
\begin{gathered}
u-\frac{2}{1+h}=-\frac{r_{0}}{a} \\
(1+h) u^{1 / 2} \cos \gamma=\sqrt{\frac{a}{r_{0}}\left(1-e^{2}\right)}
\end{gathered}
$$

where $a$ is the semi-major axis and $e$ is the eccentricity of the orbit.

Again, we neglect the aerodynamic terms to write the adjoint equations (16)

$$
\begin{aligned}
& \frac{d p_{h}}{d s}=-p_{h} \tan \gamma-\frac{2 p_{u}}{(1+h)^{2}} \tan \gamma-\frac{p_{\gamma}}{u(1+h)^{2}} \\
& \frac{d p_{u}}{d s}=-\frac{p_{\gamma}}{u^{2}(1+h)} \\
& \frac{d p_{\gamma}}{d s}=-\frac{p_{h}(1+h)}{\cos ^{2} \gamma}+\frac{2 p_{u}}{(1+h) \cos ^{2} \gamma}
\end{aligned}
$$

Using the first of the Eqs. (11) to change the independent variable from $s$ to $h$, we have

$$
\begin{aligned}
\frac{d p_{h}}{d h} & =-\frac{p_{h}}{1+h}-\frac{2 p_{u}}{(1+h)^{3}}-\frac{p_{\gamma}}{u(1+h)^{3} \tan \gamma} \\
\frac{d p_{u}}{d h} & =-\frac{p_{\gamma}}{u^{2}(1+h)^{2} \tan \gamma} \\
\frac{d p_{\gamma}}{d h} & =-\frac{p_{h}}{\sin \gamma \cos \gamma}+\frac{2 p_{u}}{(1+h)^{2} \sin \gamma \cos \gamma} .
\end{aligned}
$$


Define

$$
\begin{aligned}
c_{4}= & -p_{h}(1+h) \tan \gamma+\frac{2 p_{u}}{1+h} \tan \gamma \\
& -p_{\gamma}\left[1-\frac{1}{u(1+h)}\right] .
\end{aligned}
$$

By taking the derivative of $c_{4}$ with respect to $h$, using Eqs. (21) and the state equations without aerodynamic forces for $d u / d h$ and $d \gamma / d h$, we easily verify that $d c_{4} / d h=0$. Hence $c_{4}$ is a constant. But if $c_{0}=c_{1}=0$, and since we can always select the axis system such that $\phi=\psi=0$ at the initial time, by comparing the two equations (14) and (22), we obtain

$$
c_{4}=0
$$

Using Eqs. (22) and (23), we write the third equation of $(21)$

$$
\frac{d p_{\gamma}}{d h}=\frac{p_{\gamma}}{\sin ^{2} \gamma}\left[\frac{1}{1+h}-\frac{1}{u(1+h)^{2}}\right]
$$

With Eq. (11) for $\gamma$ without the aerodynamic term, we have the transformed equation

$$
\frac{d p_{\gamma}}{d \gamma}=\frac{p_{\gamma}}{\sin \gamma \cos \gamma}
$$

which can be integrated to give

$$
p_{\gamma}=c_{5} \tan \gamma
$$

where $c_{5}$ is a new constant. With this solution for $p_{\gamma}$, we write Eq. (21) for $p_{u}$

$$
\frac{d p_{u}}{d h}=-\frac{c_{5}}{u^{2}(1+h)^{2}}
$$

Since from the first of the integrals (19), $d u=-2 d h /(1$ $+h)^{2}$, the integral of $(27)$ is

$$
p_{u}=\frac{1}{2}\left(c_{6}-\frac{c_{5}}{u}\right)
$$

with $c_{6}$ being the last constant, completing the integration of the adjoint system.

In summary, of the adjoint system, we have 4 exact integrals and 2 approximate integrals. In the approximation we have assumed that the atmospheric forces are low enough so that along each orbital revolution, the motion is nearly Keplerian. Therefore, it is expected that the explicit optimal control laws obtained are suitable for use in the maneuver at very high altitude as in the present case.
Using the solutions (17), (26) and (28) for $p_{\psi}$, $p_{\gamma}$ and $p_{u}$ in the optimal control law (15), we have the explicit solution with the new normalized constants $k_{1}=c_{6} / c_{5}, k_{2}=c_{2} / c_{5}$ and $k_{3}=c_{3} / c_{5}$.

$$
\begin{aligned}
& C=\frac{E * \tan \gamma}{k_{1} u-1} \\
& S=\frac{E^{*}\left(k_{2} \cos \theta+k_{3} \sin \theta\right) \cos \phi}{\left(k_{1} u-1\right) \cos \gamma}
\end{aligned}
$$

The approximate control laws have been used in the exact equations of motion (11) so that we can compare their performance with the results obtained from the exact integration of the full set of state and adjoint equations (11) and (16). They provide excellent agreement as shown in Figs. 2-6. The plots show the variations of the trajectory variables $h, u, \gamma, \phi, \psi$ and $\gamma$ and also the elements of the orbit $e$ and $I$ and the controls $C$ and $S$ for a trajectory from $u_{0}=1.6$ to $u_{f}=1.0$. The value $B=0.09$ selected is low enough for the vehicle to stay in orbit for 21 revolutions. The constant $k_{i}$ are adjusted such that when $u=u_{f}$, the conditions $h_{f}=0, \gamma_{f}=0$, and the transversality condition, based on the performance (13) and the solution (17),

$$
\begin{gathered}
\tan \psi_{f}+\sin \phi_{f} \tan \left(\eta+\theta_{f}\right)=0 \\
\tan \eta=k_{2} / k_{3}
\end{gathered}
$$

are satisfied. It is found that, for a variety of initial speeds $u_{0}$ experimented, we have nearly $k_{2}=-1 / E^{*}$. Using the exact optimal controls obtained by numerical integration of the adjoint system we have $I_{f}=20.130^{\circ}$, while the suboptimal controls provide a plane change of $I_{f}=20.02^{\circ}$.

\section{Conclusions}

In this paper, we have considered the problem of using speed depletion to perform a maximum plane change in near orbital flight of a lifting hypervelocity vehicle. Besides the 4 exact integrals for the adjoint equations, we have used the fact that with low aerodynamic forces, the motion is nearly Keplerian and the remaining two equations for the adjoint system can be approximately integrated. The optimal lift and bank controls are then expressed in explicit form. The approximate controls provide excellent agreement when compared with the exact solutions. In the slow maneuver, it is seen that the orbit is slowly contracting, while there is an incremental plane change during each passage through the perigee. The orbit is slowly rotating about its apsidal line. Then when expressed in terms of 
the elements of the osculating orbit, the line of ascending node and the perigee are nearly stationary. With the explicit control laws only the state equations need be integrated. When the problem is reformulated in terms of the elements of the orbit, only the changes in the semi major axis $a$, the eccentricity $e$ and the inclination $I$ are relevant for the computation of the plane change. An accelerate technique for the computation, similar to the one used in the problem of contraction of orbit, can be implemented for a parametric evaluation of the plane change, in terms of the maximum lift-todrag ratio $E^{*}$, the initial speed $u_{0}$ and the final speed $u_{f}$. This has been carried out in Ref. 5 , and it is found that the slow maneuver provides practically the same result as that of the hard maneuver.

\section{Acknowledgement}

This work was supported by the Jet Propulsion Laboratory under contract No. 956416 with Dr. L. J. Wood as project manager.

\section{References}

1. Mease, K. D., "Optimization of Aeroassisted Orbit Transfer: Current Status." JAS, 36, 7-33 (1988).

2. Vinh, N. X. and Hanson, J. M., "Optimal Aeroassisted Return from High Earth Orbit with Plane Change," Acta Astronautica, 12 11-25 (1985).

3. Vinh, N. X., Optimal Trajectories in Atmospheric Flight, Elsevier, Amsterdam (1981).

4. Vinh, N. X. and Ma, D. M., "Optimal MultiplePass Aeroassisted Plane Change," Acta Astronautica, to appear.

5. Ma, D. M., Multiple-Pass Aeroassisted Plane Chan $-g e, \mathrm{Ph}$. D. thesis, The University of Michigan (1988).

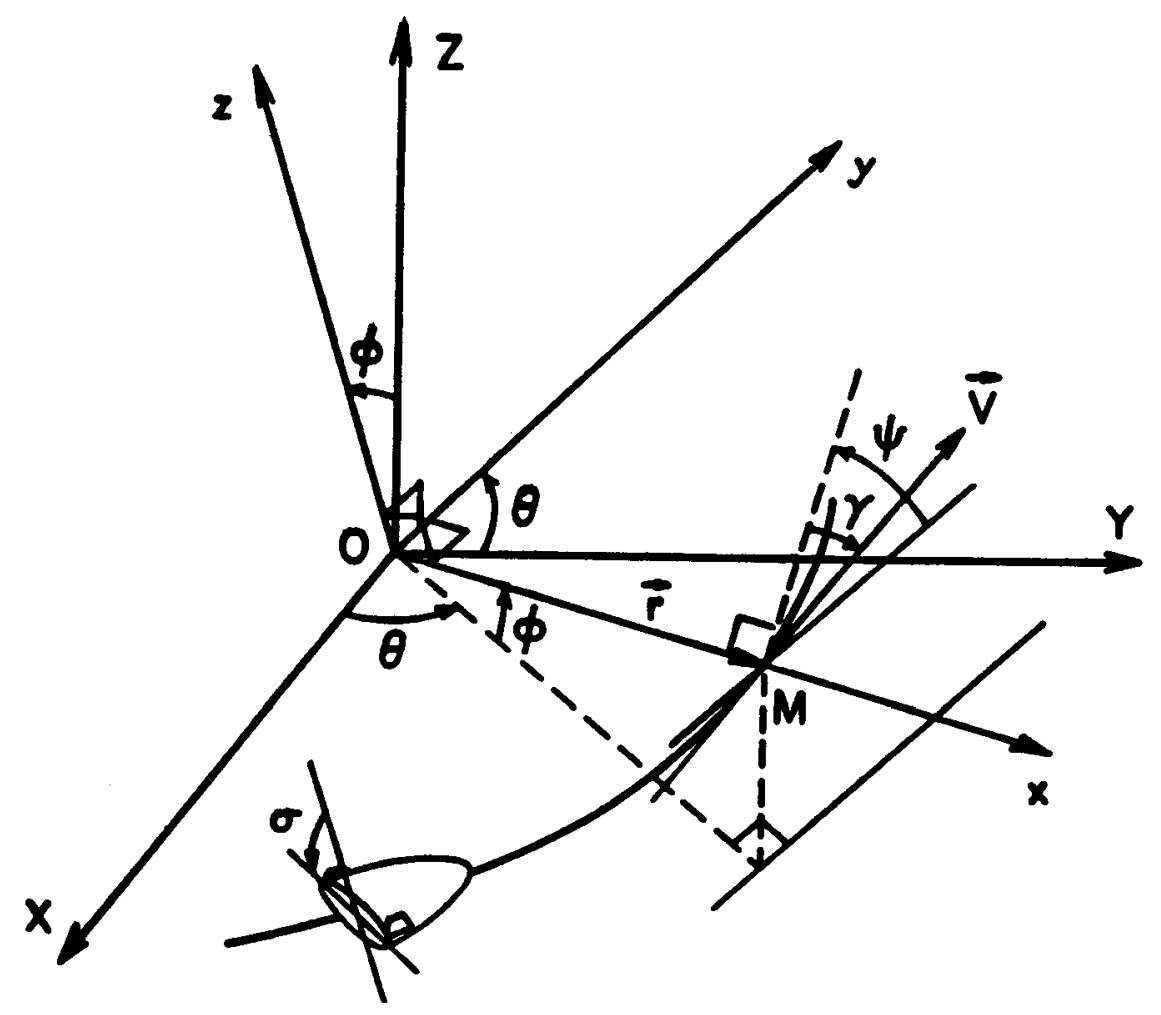

Fig. 1 Coordinate system. 

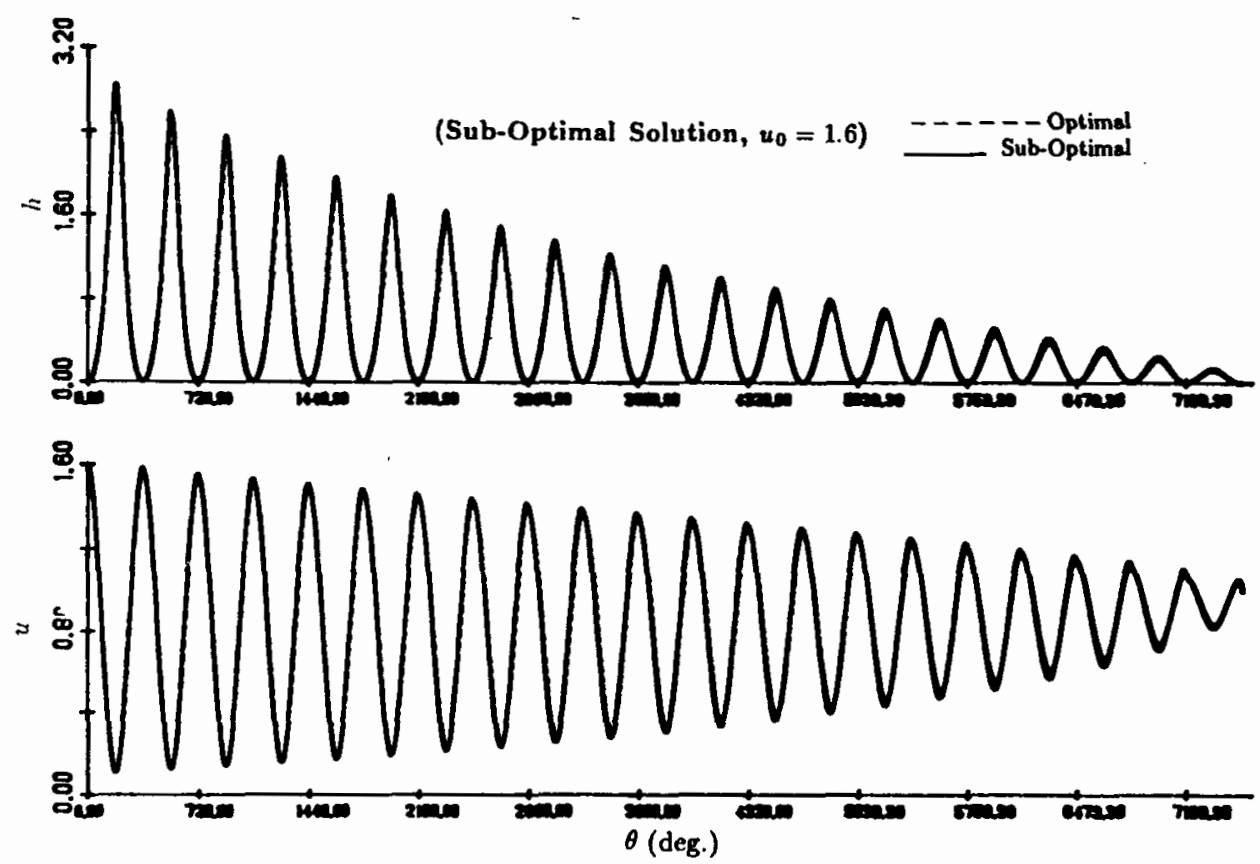

Fig. 2 The altitude and the velocity versus the longitude
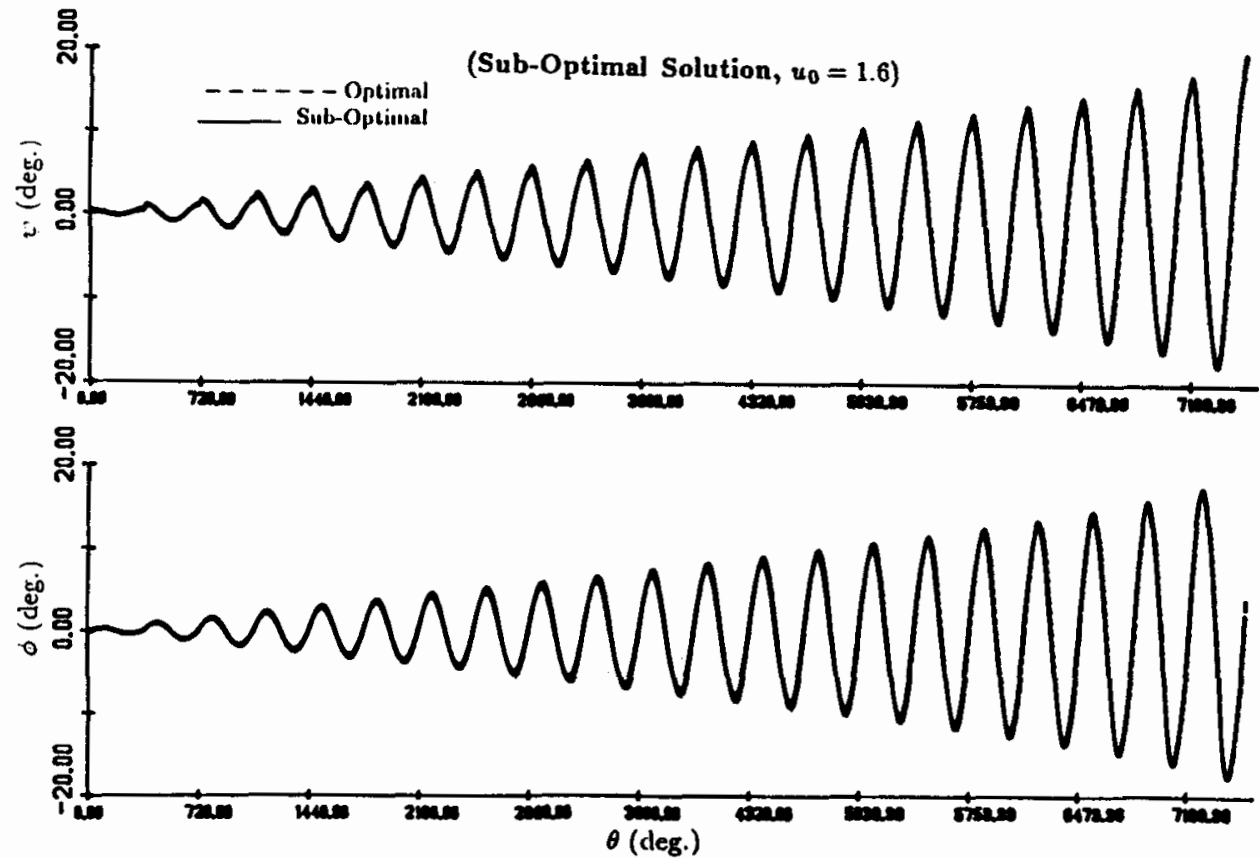

Fig. 3 The heading and the latitude versus the longitude. 


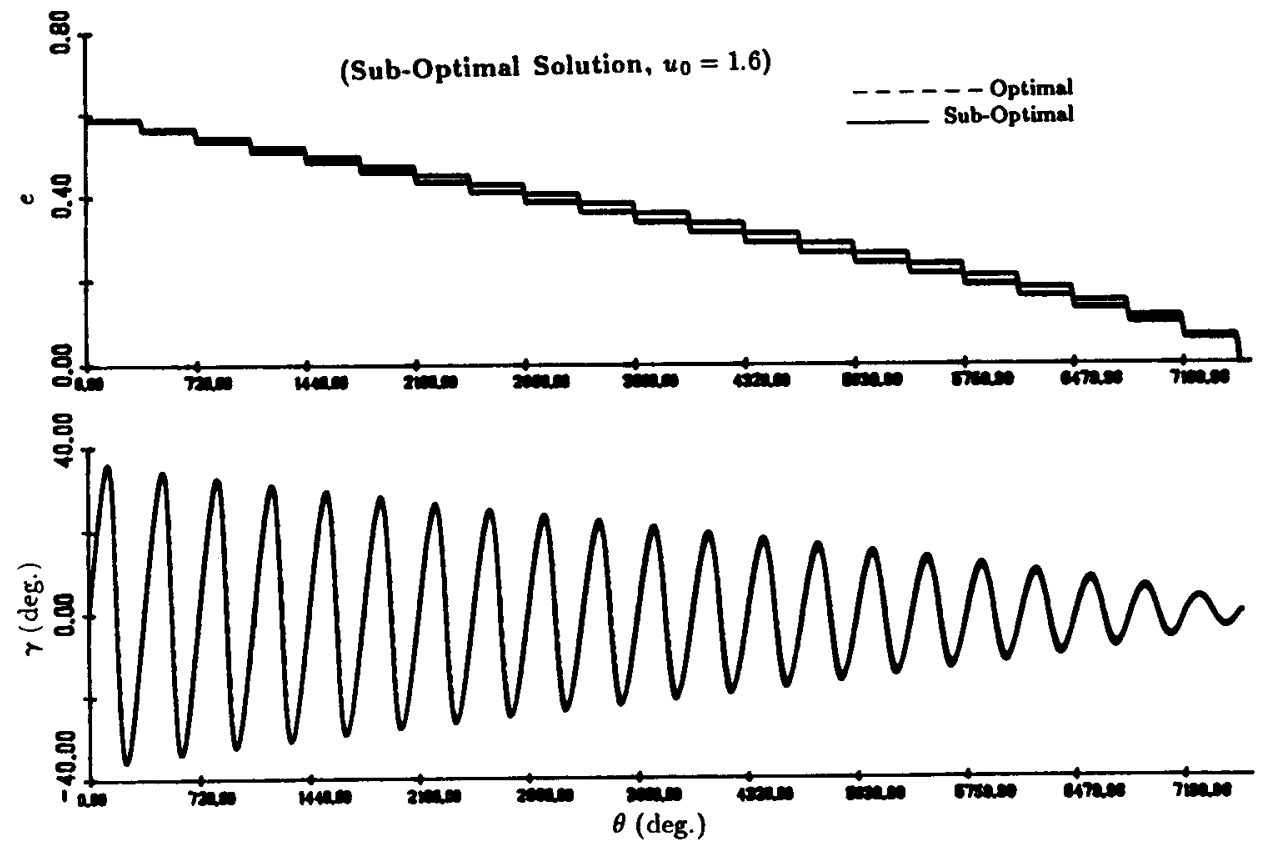

Fig. 4 The eccentricity and the flight path angle versus the longitude.
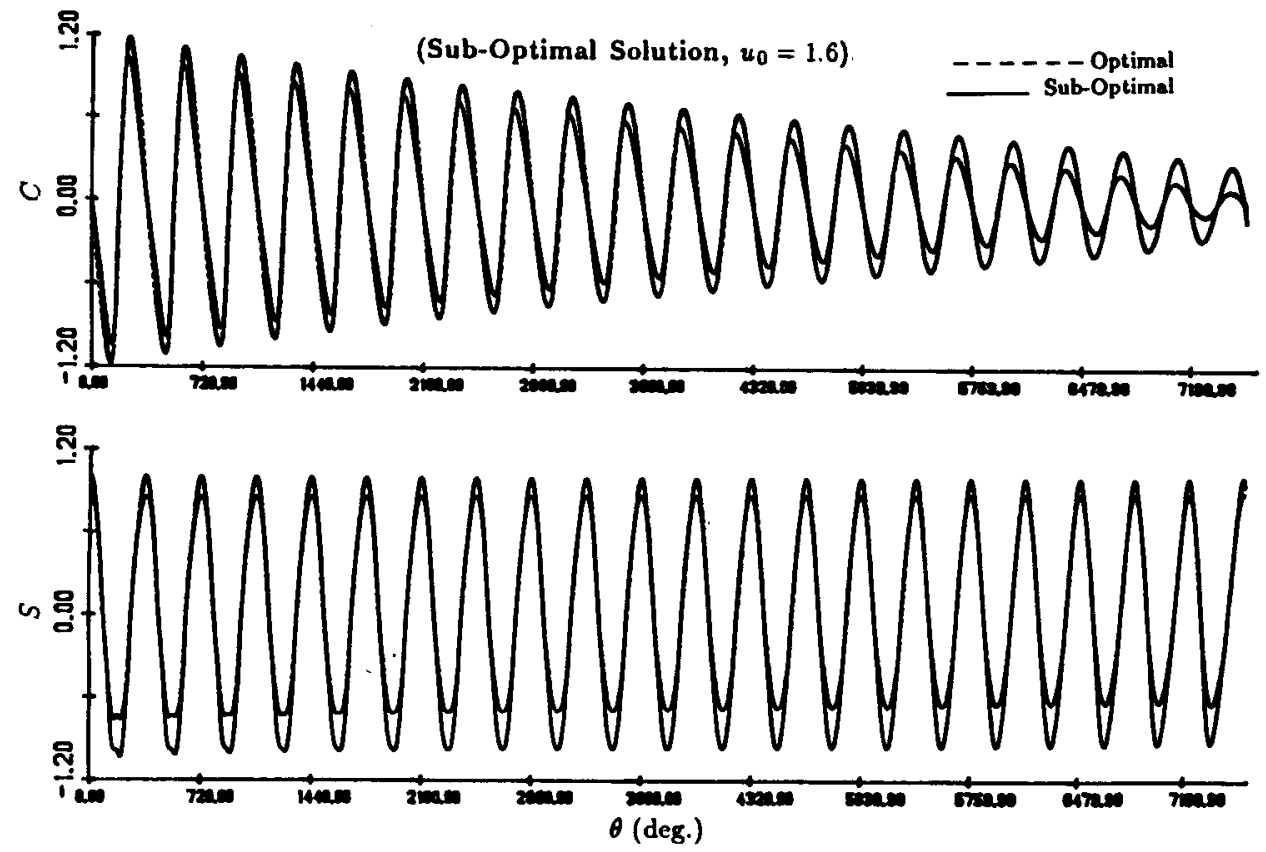

Fig. 5 The vertical and the lateral components of the normalized lift coefficient versus the longitude. 


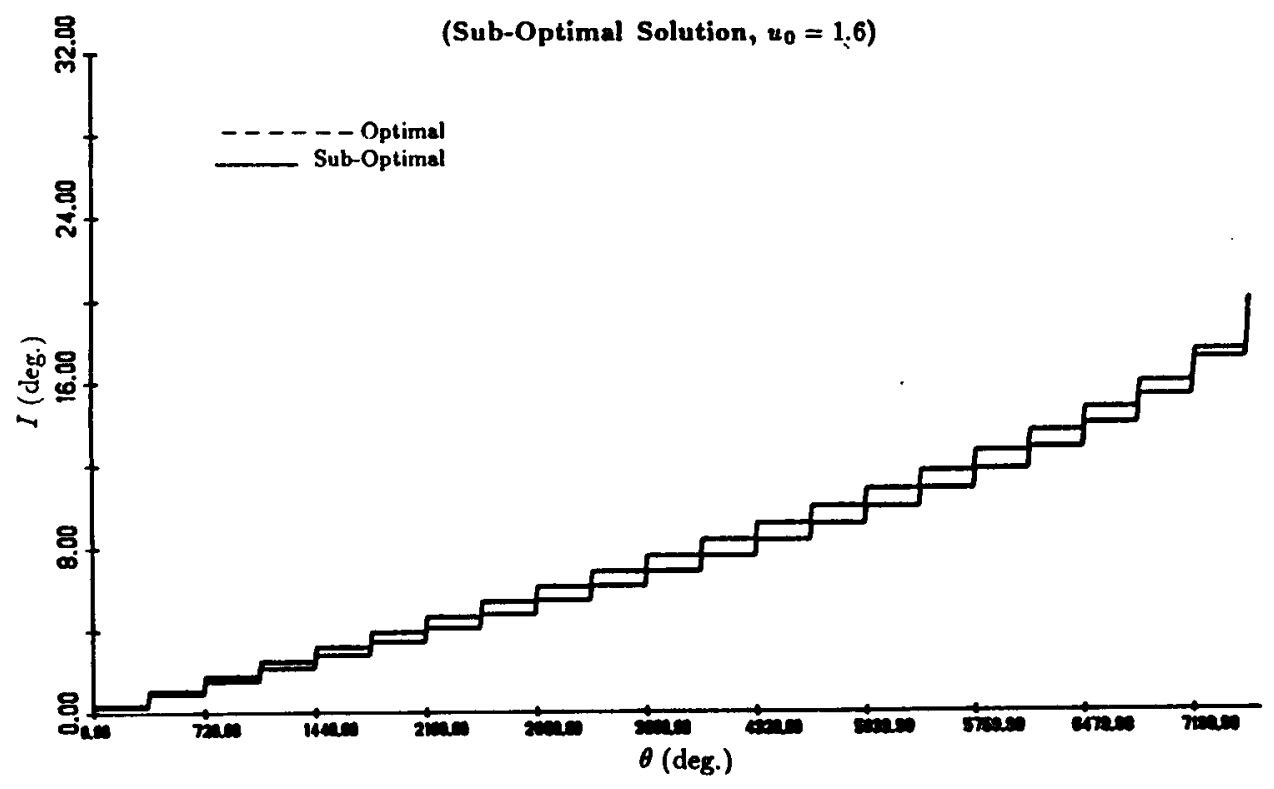

Fig. 6 The inclination angle versus the longitude. 